Journal of Advanced Computer Science \& Technology, 8 (2) (2019) 38-39
Sournal of Advanced Computer Science \& Technology
WPC
Website: $w$ ww.sciencepubco.com/index.php/JACST
Research paper

\title{
Geometric Modelling of the Thinning by Cell Complexes
}

\author{
Atefeh Hasan-Zadeh* \\ *Fouman Faculty of Engineering, College of Engineering, University of Tehran, Iran \\ E-mail: hasanzadeh.a@ut.ac.ir
}

\begin{abstract}
Motivation: Thinning is an extremely active area of research because of its primary role in reducing the amount of information that must be processed by algorithms for pattern recognition. Most thinning algorithms are supposed to be topology-preserving, although an accurate statement of what this means is usually left unanswered.

Results: The objective of this article is the presentation of a general topology via the concepts of homotopy theory to preserve the thinning. The proposed method can be applied to any decomposition of non-structural cells of the object, given that the cells have a fixed structure.
\end{abstract}

Keywords: computer vision; Thinning; Cell complex structure; Fundamental groups; Van Kampen theorem

\section{Introduction}

A skeleton is a combinatory representation of an object that preserves many of the topological and geometric properties of the original object. The process of obtaining the skeleton is usually called skeletonization or thinning. Thinning has a wide range of applications. For example it is fundamental in computer vision [1], medical imaging [2], metal molding [3], and especially digital image analysis and processing [4].

In this case, skeletons represent a simpler picture that retains the significant information of the original one. Specific applications include optical character recognition, fingerprint recognition, visual inspection, pattern recognition and matching, binary image compression and protein folding [5].

Considering the importance of reducing the amount of information to be processed by pattern recognition algorithm, in this paper, a novel approach will be examined in this regards. With a mathematical look, a geometric approach is presented to illustrate the structures and features of pattern recognition algorithms. The exposed model will be based on geometric and algebraic topology such as homotopy theory, fundamental groups and the related concepts for skeletonization or thinning [6-8].

\section{Geometric Modeling}

The reader is assumed to be familiar with the basic concepts of algebraic topology found in relevant books, including [6-8].

Theorem. A general topology-preserving method for the thinning can be obtained for any unstructured cell decomposition of the object. The proposed method is in agreement with the structure of the original objects used to image processing and the other pattern recognition algorithms.

Proof. The skeleton can be defined informally as a thinned subset of the object that retains the same topological characteristics of the original object. For the thinning usually one also requires to preserve the shape of the object, which is the main object of the successive steps below.

Step 1. Introducing Basic Concepts:

A CW complex is a space $C$ constructed in the following way:

1) Start with a discrete set $C^{0}$, the 0 -cells of $C$.

2) Inductively, form the $n$-skeleton $C^{n}$ from $C^{n-1}$ by attaching $n$-cells $c_{\alpha}^{n}$ via maps $\varphi_{\alpha}: S^{n-1} \rightarrow C^{n-1}$ from the $n-1$-dimensional sphere to $C^{n-1}$. This means that $C^{n}$ is the quotient space of $C^{n-1} \sqcup_{\alpha} D_{\alpha}^{n}$ under the identifications $x \sim \varphi_{\alpha}(x)$ for $x \in \partial D_{\alpha}^{n}$, the boundary of the $n$-dimensional disc.

3) Set $C=\cup_{n} C^{n}$ with the weak topology.

Step 2. Introducing Basic Statement:

Suppose a space $C$ is decomposed as the union of a collection of path-connected open subsets $C_{\alpha}$, each of which contains the basepoint $x_{0} \in C$. The homomorphisms $j_{\alpha}: \pi_{1}\left(C_{\alpha}\right) \rightarrow \pi_{1}(C)$ induced by the inclusions $C_{\alpha} \hookrightarrow C$ extend to a homomorphism $\Phi: *_{\alpha} \pi_{1}\left(C_{\alpha}\right) \rightarrow$

cC) (†) Copyright $\odot$ Authors. This is an open access article distributed under the Creative Commons Attribution License, which permits unrestricted use, distribution, and reproduction in any medium, provided the original work is properly cited. 
$\pi_{1}(C)$. The van Kampen theorem will say that $\Phi$ is very often surjective, but it can be expected $\Phi$ to have a nontrivial kernel in general. A full description of $\Phi$ is gained by Van Kampen's theorem:

Van Kampen Theorem [7]. If $C$ is the union of path-connected open sets $C_{\alpha}$ each containing the basepoint $x_{0} \in C$ and if each intersection $C_{\alpha} \cap C_{\beta}$ is path-connected, then the homomorphism $\Phi: *_{\alpha} \pi_{1}\left(C_{\alpha}\right) \rightarrow \pi_{1}(C)$ is surjective. If in addition each intersection $C_{\alpha} \cap C_{\beta} \cap C_{\gamma}$ is path-connected, then the kernel of $\Phi$ is the normal subgroup $N$ generated by all elements of the form $i_{\alpha \beta}(\omega) i_{\beta \alpha}(\omega)^{-1}$, where $\omega \in$ $\pi_{1}\left(C_{\alpha} \cap C_{\beta}\right)$ and $i_{\alpha \beta}: \pi_{1}\left(C_{\alpha} \cap C_{\beta}\right) \rightarrow \pi_{1}\left(C_{\alpha}\right)$ is the homomorphism induced by the inclusion $C_{\alpha} \cap C_{\beta} \hookrightarrow C_{\alpha}$, and hence $\Phi$ induces an isomorphism $\pi_{1}(C) \approx *_{\alpha} \pi_{1}\left(C_{\alpha}\right) / N$.

Step 3. Main result of the concepts and the theorem:

It is shown that the fundamental group is affected by attaching 2-cells:

Suppose a collection of 2-cells $c_{\alpha}^{2}$ has been attached to a path-connected space $C$ via maps $\varphi_{\alpha}: S^{1} \rightarrow C$, producing a space $E$. If $s_{0}$ is a basepoint of $S^{1}$ then $\varphi_{\alpha}$ determines a loop at $\varphi_{\alpha}\left(s_{0}\right)$ that will be called $\varphi_{\alpha}$, even though technically loops are maps $I \rightarrow C$ rather than $S^{1} \rightarrow C$. For different $\alpha$ 's the basepoints $\varphi_{\alpha}\left(s_{0}\right)$ of these loops $\varphi_{\alpha}$ may not all coincide.

To remedy this, choose a basepoint $x_{0} \in C$ and a path $\gamma_{\alpha}$ in $C$ from $x_{0}$ to $\varphi_{\alpha}\left(s_{0}\right)$ for each $\alpha$. Then $\gamma_{\alpha} \varphi_{\alpha} \overline{\gamma_{\alpha}}$ is a loop at $x_{0}$. This loop may not be nullhomotopic in $C$, but it will certainly be nullhomotopic after the cell $c_{\alpha}^{2}$ is attached. Thus the normal subgroup $N \subset \pi_{1}\left(C, x_{0}\right)$ generated by all the loops $\gamma_{\alpha} \varphi_{\alpha} \overline{\gamma_{\alpha}}$ for varying $\alpha$ lies in the kernel of the map $\pi_{1}\left(C, x_{0}\right) \rightarrow \pi_{1}\left(E, x_{0}\right)$ induced by the inclusion $C \hookrightarrow E$. If $E$ is obtained from $C$ by attaching 2-cells as described above, then the inclusion $C \hookrightarrow E$ induces a surjectionwhose kernel is $N$. Thus $\pi_{1}(E) \approx \pi_{1}(C) / N$.

If $E$ is obtained from $C$ by attaching $n$-cells for a fixed $n>2$, then the inclusion $C \hookrightarrow E$ induces an isomorphism $\pi_{1}\left(C, x_{0}\right) \approx \pi_{1}\left(E, x_{0}\right)$. For a path-connected cell complex $C$ the inclusion of the 2-skeleton $C^{2} \hookrightarrow C$ induces an isomorphism $\pi_{1}\left(C^{2}, x_{0}\right) \approx \pi_{1}\left(C, x_{0}\right)$.

\section{Conclusion}

In this paper a general topology-preserving method for the thinning was introduced. The presented topology can be used for any unstructured cell decomposition of the object. Since, it is in agreement with the structure of thinning, it can be propose a universal law for the reduction of the amount of information to be processed by pattern recognition algorithms.

\section{Acknowledgement}

This paper is supported by Fouman Faculty of Engineering, College of Engineering, University of Tehran.

\section{References}

[1] Tatt TK, Muhamad N, Che Haron CH, Jamaludin KR, "Influences of Injection Pressure and Flow Rate to the Green Properties”, International Journal of Engineering \& Technology, Vol. 8, No. 1.12, (2019), pp. 51-54.

[2] Schaap M, Metz CT, van Walsum T, van der Giessen AG, Weustink AC, Mollet NR, Bauer C, Bogunović H, Castro C, Deng X, Dikicih E, O'Donnell T, Frenay M, Friman O, Hoyos MH, Kitslaar PH, Krissian K, Kühnel C \& Niessen WJ, "Standardized evaluation methodology and reference database for evaluating coronary artery centerline extraction algorithms”, Medical Image Analysis, Vol. 13, No. 5, (2009), pp. 701-714.

[3] Maskara SL, "Computer Vision, Artificial Intelligence and Robotics - A cursory Overview of Some of the Wonders of Modern Science and Technology", Conference: National Seminar on Computer Vision \& Image Processing, 2017, DOI:10.13140/RG.2.2.14488.90886.

[4] Lam L, Lee SW \& Suen SY, "Thinning Methodologies-A Comprehensive Survey", IEEE Transactions on Pattern Analysis and Machine Intelligence, Vol. 14, No. 9, (1992), pp. 869-885.

[5] Abeysinghe S, Ju T, Baker ML \& Chiu W, "Shape modeling and matching in identifying 3D protein structures", Computer-Aided Design, Vol. 40, No. 6, (1992), pp. 708-720.

[6] Milnor J, "Topology from the Differentiable Viewpoint", Princeton University Press, 1997.

[7] Bredon G, "Topology and Geometry", Springe, 1993.

[8] Fulton W, “Algebraic Topology: A First Course”, Springer, 1995. 